\title{
Médiévales
}

Langues, Textes, Histoire

71 | automne 2016

Conflits et concurrence de normes

\section{Conflits de normes dans le débat scolastique sur le baptême forcé des enfants juifs (XIII ${ }^{\mathrm{e}} \mathrm{XIV}^{\mathrm{e}}$ siècle)}

Conflicts of Norms in Scholastic Debates on Forced Baptism of Jewish Children (Thirteenth-Fourteenth Century)

\section{Elsa Marmursztejn}

\section{OpenEdition}

\section{Journals}

Édition électronique

URL : https://journals.openedition.org/medievales/7890

DOI : 10.4000/medievales.7890

ISSN : 1777-5892

Éditeur

Presses universitaires de Vincennes

Édition imprimée

Date de publication : 20 novembre 2016

Pagination : $27-40$

ISBN : 978-2-84292-565-9

ISSN : 0751-2708

Référence électronique

Elsa Marmursztejn, "Conflits de normes dans le débat scolastique sur le baptême forcé des enfants juifs (xIII-xIv ${ }^{\mathrm{e}}$ siècle) », Médiévales [En ligne], 71 | automne 2016, mis en ligne le 20 novembre 2018 consulté le 24 avril 2022. URL : http://journals.openedition.org/medievales/7890 ; DOI : https:// doi.org/10.4000/medievales.7890 


\title{
Conflits de normes dans le débat scolastique sur le baptême forcé des enfants juifs (XIII ${ }^{\mathrm{e}}$-XIV ${ }^{\mathrm{e}}$ siècle)
}

\begin{abstract}
À partir du XIII ${ }^{\mathrm{e}}$ siècle, l'Université de Paris compte au nombre des multiples succursales médiévales de la « fabrique de la norme ${ }^{1}$ ». Dès avant l'exclusion des études juridiques, en $1219^{2}$, le projet pontifical de faire de cette université l'instance suprême de la chrétienté latine en matière d'orthodoxie doctrinale avait conforté sa spécialisation théologique. La faculté de théologie forme dès lors le pôle majeur de la production de normes rationnelles, répondant au programme de construction de la vérité par l'enquête formulé par Abélard vers 1130. Fruits de la raison scolastique, ces normes ne se cantonnent pas dans le champ spéculatif. Elles s'appliquent aussi bien aux situations communes et aux questions pratiques que leurs cadres d'énonciation apparentent aux casus juridiques. Au-delà de cette similitude formelle, la teneur même du raisonnement théologique révèle une interaction forte avec le droit, capté et dépassé. C'est en effet en fondant leurs solutions sur la norme suprajuridique du droit divin ou naturel que les théologiens ont revendiqué la suprématie normative de leur discipline. Si elle s'élabore sur un socle de références communes, fortement unifiées par les savoirs religieux, la normativité théologique n'exclut pas les dissonances. La foi repose sur les fondements stables de l'Écriture, mais la théologie, subalternée à la science de Dieu, admet les divergences dans
\end{abstract}

1. $C f$. E. Marmursztejn, «Une fabrique de la norme au XiII siècle : l'université de Paris », dans V. Beaulande, J. Claustre et E. Marmursztejn éd., La Fabrique de la norme. Lieux et modes de production des normes au Moyen Âge et à l'époque moderne, Rennes, 2012, p. 31-48.

2. Par la bulle Super speculam, promulguée le 16 novembre 1219 par le pape Honorius III, qui interdit l'enseignement du droit romain à Paris (Chartularium Universitatis Parisiensis, H. Denifle et E. Châtelain éd., Paris, 1889, vol. 1, nº 32, p. 92). 
l'expression des opinions humaines ${ }^{3}$. Certains maîtres ont d'ailleurs exalté les vertus heuristiques de la libre confrontation des opinions, qui permettait de parvenir à la position la plus conforme à la raison sur des sujets dont la vérité n'était pas déterminée de façon absolument évidente ${ }^{4}$. La vérité se construisait dans la concurrence, voire dans le choc des opinions.

C'est précisément dans une optique focalisée sur les formes et sur la productivité des tensions, de la concurrence et des conflits entre instances et références normatives, que l'on voudrait ici envisager la question précise du baptême forcé des enfants juifs, c'est-à-dire, en termes exacts, la question de savoir s'il fallait baptiser les enfants des juifs contre la volonté de leurs parents, telle qu'elle a été successivement traitée, entre 1269 et 1340, par le dominicain Thomas d'Aquin ${ }^{5}$, les franciscains Richard de Mediavilla ${ }^{6}$ et Jean Duns $\operatorname{Scot}^{7}$, les dominicains Durand de Saint-Pourçain ${ }^{8}$ et Pierre de la Palud ${ }^{9}$, le carme catalan Guido Terreni ${ }^{10}$. Cette question n'est pas, comme on pourrait le croire, la simple mise en scène d'une bataille gagnée d'avance, destinée à proclamer, sous la pression de la contrainte dogmatique ou du conformisme doctrinal, la victoire attendue des normes salvifiques de la «Loi nouvelle ». Non seulement la question a donné lieu à une authentique controverse, mais elle ne s'est pas portée sur le terrain « légal » de l'opposition entre christianisme et judaïsme. Elle a été envisagée comme un conflit de droits sur l'enfant, dont la résolution impliquait de déterminer les droits qui s'exerçaient sur les juifs. La réflexion scolastique prend consistance dans un contexte où les politiques de conversion des juifs participent à la définition plus stricte des monarchies occidentales comme

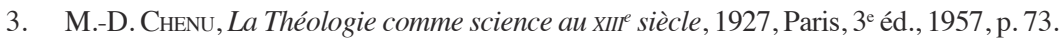

4. Cf. Godefroid de Fontaines, Quodlibet XII, 5 (H. HofFmans éd., Louvain, 1932, p. 101).

5. Thomas D'AQuin, Quodlibet I, 7 : « Utrum parvuli Iudeorum sint baptizandi invitis parentibus » (éd. R.-A. GAUTHIER, Quaestiones de quolibet, Rome, Édition léonine, t. XXV, 1996, vol. 2, p. 221-224) ; Summa theologiae, IIa IIae, q. 10, a. 12 : «Utrum pueri iudaeorum et aliorum infidelium sint invitis parentibus baptizandi » (Rome, Édition léonine, t. VIII, 1895, p. 93-95) ; Summa theologiae, IIIa, q. 68, a. 10 : «Utrum pueri iudaeorum vel aliorum infidelium sint baptizandi etiam invitis parentibus » (Rome, Édition léonine, t. XII, 1906, p. 102-103).

6. Richard de Mediavilla, In IV Sent., D. 6, a. 3, q. 3 : « Utrum parvuli Iudaeorum et Paganorum sint invitis parentibus baptizandi » (Brescia, 1591 ; repr. Francfort, 1963, p. 78-79).

7. Duns Scot, Ordinatio in IV Sent., Opus oxoniense, D. 4, q. 9: « De parvulis Iudeorum et infidelium, an sint invitis parentibus baptizandi » (éd. E. MARMURszTEJn et S. PIRON, « Duns Scot et la politique. Pouvoir du prince et conversion des juifs », dans O. Boulnois et al. éd., Duns Scot à Paris. 1302-2002, Turnhout, p. 58-62).

8. Durand de Saint-Pourçain, In IV Sent., lib. 4, D. 4, q. 6 : «Utrum filii infidelium debeant baptizari invitis parentibus eorum » (Lyon, 1563, f. 259va-260rb).

9. Pierre de la Palud, In IV Sent., D. 4, q. 4, a. 1 : « De carentibus usu rationis, utrum sint baptizandi » (Venise, 1493, f. 19vb-21rb).

10. Guido Terreni, Apparatus in Decretum Gratiani, De Consecratione, D. 4, c. 110 : «Utrum parvuli Iudaeorum sint invitis parentibus baptizandi » (Paris, BnF, ms. lat. 3914, f. 327r-330r). 
monarchies chrétiennes et où la revendication royale d'une juridiction exclusive sur les juifs se présente comme un aspect de l'affirmation générale de la souveraineté monarchique.

À divers égards, la question singulière du baptême forcé des enfants juifs est propice à une réflexion sur les conflits de normes : d'abord, parce que ces conflits présentent différents niveaux de pertinence dans le champ scolastique ; ensuite, parce que la spécificité de la question a mis au centre de la discussion des enjeux politiques et juridiques a priori inattendus ; enfin, parce que le traitement même de la question a produit différentes figures du conflit majeur entre droits des parents juifs et pouvoir du prince chrétien.

\section{La pertinence scolastique des conflits de normes}

À un premier niveau, le conflit de normes apparaît comme le moteur même de l'enquête scolastique. De façon générale, le savoir chrétien s'est élaboré par l'élucidation de l'Écriture et par l'explicitation des normes que n'avait pas déterminées la loi évangélique, conçue, par opposition à la Loi ancienne, comme « loi de grâce » ou « loi de liberté » ${ }^{11}$. Le rôle spécifique des théologiens scolastiques tient à une mutation majeure du régime d'accession à la vérité ${ }^{12}$. Il s'agissait, suivant les termes du prologue du Sic et non d'Abélard, de construire la vérité par l'enquête, en confrontant les autorités dissonantes ${ }^{13}$. Le Sic et non se présente du reste comme un volumineux recueil des opinions contradictoires des Pères de l'Église, rassemblées en questions. La quaestio, qui forme depuis le XIII ${ }^{\mathrm{e}}$ siècle l'unité de réflexion de tous les genres de la scolastique ${ }^{14}$ - et qui fonctionne donc de façon identique dans les Quodlibets et dans la Somme de théologie de Thomas d'Aquin, dans les commentaires sur les Sentences de Richard de Mediavilla, Duns Scot, Durand de Saint-Pourçain ou Pierre de la Palud, et dans le commentaire de Guido Terreni sur le Décret de Gratien ${ }^{15}$-,

11. Je me permets de renvoyer sur ce point à E. MARMurszTEjn, « Loi ancienne, loi nouvelle et normes chrétiennes dans la théologie scolastique du XIII ${ }^{\mathrm{e}}$ siècle », Revue de l'histoire des religions, 228 (2011), p. 509-539.

12. Cf. A. Boureau, «Droit et théologie au XIII e siècle », Annales ESC, 47/6 (1992), p. 1113-1125.

13. Cf. Pierre Abélard, Sic et non, éd. B. R. Boyer et R. McKeon, Chicago/Londres, 1976-1977, p. 103.

14. Cf. B. C. Bazan et al., Les Questions disputées et les questions quodlibétiques dans les facultés de théologie, de droit et de médecine, Turnhout, 1985.

15. Le carme Guido Terreni, maître en théologie à Paris avant d'être élu prieur de son ordre en 1318, évêque de Majorque en 1321, puis d'Elne en 1322, est le premier théologien à avoir commenté le Décret de Gratien avant Jean de Torquemada au milieu du Xv siècle. Son projet consistait à livrer une interprétation des textes du Décret qui intéressaient directement les théologiens. Cf. Th. Turley, « Guido Terreni and the Decretum », Bulletin of Medieval Canon Law, 8 (1978), p. 29-34. 
représente l'expression achevée du programme d'Abélard. La construction rationnelle des normes chrétiennes s'est faite dans le conflit.

En outre, dans le secteur de la morale pratique, les théologiens des $\mathrm{XIII}^{\mathrm{e}}$ et XIV $\mathrm{X}^{\mathrm{e}}$ siècles ont systématiquement traduit en conflits de normes les cas qu'ils traitaient et, de façon générale, fondé la légitimité normative de leur propre discours sur le fondement suprajuridique du « droit divin ou naturel ${ }^{16}$. Yan Thomas a souligné que la position de la nature avait changé au Bas-Empire. La loi naturelle du christianisme tardo-antique avait transporté dans la nature, créée par le législateur divin, les interdits que le droit romain n'attachait qu'à la loi humaine. Comme les Pères de l'Église, le droit de Justinien postule une équivalence entre la loi divine révélée et le droit naturel inscrit dans le cœur des hommes, selon la formulation paulinienne de la loi morale universelle, et fermement imprimé dans leur raison, suivant la glose ${ }^{17}$. Ce rapprochement entre nature et volonté divine a trouvé sa pleine expression dans la formule Natura, id est Deus, répandue dès les premiers glossateurs du droit romain et notamment employée, dans le domaine théologique, par Abélard ${ }^{18}$. Dans le cadre de cette assimilation entre Dieu et une nature édictrice de normes, les théologiens scolastiques ont revendiqué une compétence propre et une aptitude supérieure à déterminer les préceptes seconds du droit naturel, c'est-à-dire les préceptes qui étaient susceptibles de varier en fonction des situations particulières et qu'il fallait construire par le raisonnement.

Aussi la normativité théologique n'exclut-elle pas les divergences, admises et revendiquées par les maîtres. Les formes prises par cette aspiration à l'indépendance intellectuelle n'ont rien d'abstrait. Non seulement les maîtres ont défendu le programme d'Abélard en arguant des vertus heuristiques de la confrontation des opinions et en revendiquant leur libre expression comme la condition même de la recherche de la vérité, non seulement ils l'ont illustré en appliquant, dans leur propre travail, la méthode scolastique de l'enquête contradictoire, mais ils se sont euxmêmes engagés dans des débats réels, que la lecture croisée de leurs textes permet de reconstituer. La controverse scolastique apparaît ainsi comme le second niveau de pertinence du conflit de normes. La question du baptême forcé $\mathrm{a}$, de fait, donné lieu à des divergences radicales. On verra que la ligne thomiste, hostile au baptême forcé des enfants juifs, est suivie par Richard de Mediavilla, dont Duns Scot conteste directement la solution ; que Durand

16. Je me permets de renvoyer sur ce point à E. Marmursztejn, L'Autorité des maitres. Scolastique, normes et société au XIII siècle, Paris, 2007.

17. Y. Tномаs, «Imago naturae. Note sur l'institutionnalité de la nature à Rome », dans Théologie et droit dans la formation de l'État moderne, Rome/Paris, 1991 (EFR 147), p. 208 et n. 25 .

18. $C f$. J. Chiffoleau, « Contra naturam. Pour une approche casuistique et procédurale de la nature médiévale », Micrologus, 4 (1996), p. 271. 
de Saint-Pourçain rejoint la position de Duns Scot en faveur du baptême forcé des enfants juifs, mais par de tout autres voies ; que Pierre de la Palud confronte les opinions de Thomas d'Aquin et de Durand de Saint-Pourçain et tranche en faveur de Thomas, comme le fera aussi Guido Terreni, qui s'inspire de Thomas et de Pierre de la Palud pour réfuter l'opinion scotiste. Les liens entre les textes font ainsi apparaître un authentique débat qui se cristallise, au tournant du XIII ${ }^{\mathrm{e}}$ et du XIV ${ }^{\mathrm{e}}$ siècle, dans l'opposition des opinions de Thomas d'Aquin et de Duns Scot.

Les conflits de normes présentent ainsi deux niveaux de pertinence dans le champ scolastique : celui de la pratique singulière de la méthode, c'est-à-dire de la construction de l'enquête contradictoire propre à chaque auteur ; celui de la pratique collective du débat entre théologiens considérés comme instances normatives. Il faut approcher de plus près la question du baptême forcé des enfants juifs pour observer à quel niveau les scolastiques ont situé le conflit de normes.

\section{L'origine d'un conflit de droits : le cas limite des enfants}

La formulation même de la question oppose la volonté des parents juifs à une norme universelle de la loi nouvelle, qui prescrit le sacrement de baptême comme unique remède efficace au péché originel et comme condition sine qua non du salut ${ }^{19}$. Cette opposition prend sens dans le cadre de la doctrine qui a construit la nécessité du baptême sur le terrain de l'histoire du salut, par analogie et par écart avec le précepte de la circoncision, dans le cadre général de l'opposition entre loi évangélique et Loi ancienne. Comme le dit Pierre Lombard, la circoncision avait remédié au péché originel jusqu'à ce que la mort du Christ mette un terme aux legalia. La circoncision était alors devenue non seulement inutile, mais nuisible ${ }^{20}$. Thomas d'Aquin, comme Duns Scot, la conçoivent comme la préfiguration du baptême. Elle faisait partie de ces préceptes cérémoniels qui ne demeuraient pas en eux-mêmes, mais seulement dans ce qu'ils signifiaient. En l'espèce, la purification du péché constituait la condition du salut. À l'instar de la Loi ancienne elle-même, la circoncision avait été accomplie et parachevée par le baptême, qui était commun à tous les sexes et à tous les âges, conférait une grâce plus abondante et ouvrait les portes du royaume des cieux ${ }^{21}$. Le conflit de normes religieuses était ainsi résolu par la captation typologique de la Loi ancienne par la nouvelle Loi.

Les théologiens n'ont toutefois pas traité la question sur les baptêmes forcés sur le terrain « légal » de l'opposition entre christianisme et judaïsme. L'obsolescence des legalia et la nécessité du sacrement de baptême sont

19. $C f$. Jn 3,5 et Mt 28,19 .

20. Pierre Lombard, Sententiae in IV libris distinctae (Grottaferrata, 1981, t. II, p. 250).

21. Cf. Jean Duns Scot, In IV Sententiarum, D. 3, q. 4 (Lyon, 1634, p. 189). 
présupposées. L'interrogation porte sur un aspect de la coexistence concrète entre juifs et chrétiens. Elle se présente comme une expression de la tension inhérente à la doctrine d'origine augustinienne qui justifiait la survivance du judaïsme en régime chrétien. Cette doctrine se résume alors à trois arguments communs : les juifs déicides devaient être conservés pour être punis de leur crime, comme peuple témoin de la vérité du christianisme et en vue de la conversion finale de leur $«$ reste ${ }^{22}$. La doctrine de justification de la présence juive était en outre confortée, depuis le XII siècle, par la bulle de protection Sicut Iudeis, initialement accordée par le pape Calixte II en 1122 ou 1123 et reprise par vingt-trois papes entre le XII et le XVe siècle sans modifications substantielles ${ }^{23}$.

Le cas limite des enfants constitue la spécificité majeure de la question. Les baptêmes forcés d'adultes étaient en effet explicitement interdits par le droit canon. À la suite de la conversion générale des juifs du royaume wisigothique, ordonnée par Sisebut vers 615, le canon De Iudeis du concile de Tolède $\mathrm{IV}^{24}$ (633) avait interdit que les juifs fussent désormais baptisés contre leur volonté, tout en contraignant les juifs baptisés de force à observer la foi reçue. Ce canon avait été relayé en diverses collections canoniques et inséré au Décret de Gratien ${ }^{25}$ avant le milieu du XII ${ }^{\mathrm{e}}$ siècle. Dans ce cadre juridique, les jeunes enfants, jugés constitutivement incapables d'exercer leur raison et leur volonté, représentaient un cas limite dans la mesure où, à la différence des adultes, ils ne pouvaient ni consentir au baptême ni le refuser. En outre, l'éducation pouvait se substituer, dans leur cas, à la contrainte, à condition qu'ils soient enlevés à leurs parents et confiés à des familles ou à des institutions chrétiennes, suivant les termes d'un autre canon du concile de Tolède IV inséré au Décret de Gratien (Iudeorum $)^{26}$. Ce canon n'est jamais invoqué dans la discussion, mais le postulat, commun aux auteurs scolastiques, du danger qu'il y aurait à laisser les enfants baptisés aux soins de leurs parents juifs, met la licéité du rapt au cœur du débat. L'enfant représente dès lors l'enjeu d'un conflit de droits, dont la résolution impliquait de définir la nature et la mesure du pouvoir que le prince exerçait sur les juifs. Ce conflit de droits se décline de trois

22. Cf. G. Dahan, Les Intellectuels chrétiens et les juifs au Moyen Âge, Paris, 1990, p. 573-581.

23. Cf. S. Grayzel, «The Papal Bull Sicut Iudeis », dans M. Ben-Horin, B. D. Weinry et al. éd., Studies and Essays in Honor of Abraham A. Neuman, Leyde, 1962, p. 243-280, repris dans J. CoHEN éd., Essential Papers on Judaism and Christianity in Conflict. From Late Antiquity to the Reformation, New York, 1991, p. 231-259.

24. Concile de Tolède IV, canon 57 (G. Martinez Diez et F. Rodriguez éd., La colección canónica hispana, t. V, Concilios hispanos, segunda parte, Madrid, 1992, p. 235-236).

25. Décret de Gratien, D. 45, c. 5 (E. FriedBerg éd., Decretum Gratiani, Leipzig, 1879, t. I, col. 161-162).

26. Concile de Tolède IV, canon 60, éd. cit., p. 238 ; Décret de Gratien, C. 28, q. 1, c. 11, éd. cit. col. 1087. 
manières : le droit du prince est opposé au droit des parents en tant que tels (chez Thomas d'Aquin et Richard de Mediavilla), en tant que sujets (chez Duns Scot), en tant qu'esclaves (chez Durand de Saint-Pourçain, Pierre de la Palud et Guido Terreni).

\section{Le conflit entre droit du prince et droit des parents}

C'est Thomas d'Aquin qui introduit la question du baptême forcé des enfants juifs dans le débat universitaire. Il en traite à trois reprises, d'abord dans son Quodlibet de 1269, puis dans sa Somme de théologie au début des années 1270. Il constitue l'essentiel du stock des arguments utilisés par la suite. La position qu'il adopte sur la question du baptême forcé des enfants tranche sur l'évolution générale de la condition des juifs ${ }^{27}$.

Aux arguments du risque de mort éternelle encouru par les enfants, de la servitude des juifs qui donnait aux princes tout pouvoir sur leurs enfants, et du fait qu'un enfant appartenait davantage à Dieu, dont il tenait son âme, qu'aux parents dont il tenait son corps, Thomas d'Aquin oppose un unique argument contra, qu'il emprunte au décrétiste Huguccio : il ne fallait faire injustice à personne (Nemini facienda est iniuria). Or on ferait injustice aux juifs en baptisant leurs enfants contre leur gré ${ }^{28}$, parce qu'on les priverait de leur droit de puissance paternelle sur des enfants devenus chrétiens ${ }^{29}$. La patria potestas semble donner consistance juridique à la volonté des parents juifs : la norme religieuse entre ici en conflit avec le droit des parents.

La responsio de Thomas d'Aquin s'inscrit dans la perspective ouverte par Huguccio. À la norme universelle du baptême, le dominicain oppose en premier lieu, non pas la patria potestas, dont il n'est plus question, mais la « coutume de l'Église » (consuetudo ecclesie) ${ }^{30}$ : même à l'époque des puissants princes catholiques Constantin et Théodose, l'Église n'avait pas eu pour usage de baptiser les enfants juifs contre le gré de leurs parents, ce que les grands évêques Sylvestre et Ambroise, qui conseillaient respectivement ces princes, n'auraient pourtant pas manqué de leur recommander si cela avait été «conforme à la raison» (consonum rationi) ${ }^{31}$. Deux arguments soutiennent la rationalité de la coutume de l'Église : celui du péril pour la foi, car les enfants baptisés laissés à leurs parents seraient facilement détournés du christianisme, et celui de la justice naturelle, qui interdisait d'enlever les jeunes enfants à leurs parents. L'autorité d'Aristote étaye l'argument de la justice naturelle : dans l'Éthique à Nicomaque, le philosophe affirmait

27. Cf. J. Le Goff, Saint Louis, Paris, 1996, p. 793-814.

28. Huguccio, Summa super Decretum, glose sur le canon « Iudeorum » du Décret de Gratien, C. 28, q. 1, c. 11 (Paris, BnF, ms. lat. 3892, f. 296r).

29. Thomas D’Aquin, Quodlibet II, 7, p. 223.

30. Ibid.

31. Ibid. 
que l'enfant était «naturellement quelque chose de son parent ${ }^{32} »$. Thomas d'Aquin considérait que ce régime initial d'indistinction corporelle se prolongeait en un régime d'indistinction spirituelle : l'enfant se trouvait « sous la tutelle de ses parents comme en un sein spirituel » (sub parentum cura sicut sub quodam spirituali utero ${ }^{33}$ jusqu'à ce qu'il ait acquis l'usage de la raison. Tant qu'il en était dépourvu, il ne différait pas d'un « animal irrationnel » et, de même que le propriétaire d'un bœuf ou d'un cheval pouvait, selon le droit civil, utiliser sa bête à sa guise, " comme son instrument propre », l'enfant se trouvait, selon le droit naturel, sous la tutelle de son père ${ }^{34}$. Appréhendé dans l'absolu (car il n'est jamais spécifiquement question des parents juifs), référé dans les arguments à la patria potestas, conforme à la justice naturelle et comparable à un droit de propriété, le droit des parents l'emporte ainsi, de façon tout à fait surprenante, sur le salut des enfants. Thomas d'Aquin produit toutefois une norme provisoire. Lorsque l'enfant était devenu capable de faire usage de son libre arbitre, il commençait à s'appartenir et pouvait « pourvoir pour lui-même aux choses qui relèvent du droit divin ou naturel ». Il fallait alors « l'amener à la foi, non par la contrainte, mais par la persuasion » et l'enfant pouvait, « même contre le gré de ses parents, consentir à la foi et être baptisé » ${ }^{35}$.

En définissant la tutelle des parents sur leurs jeunes enfants comme un droit de propriété inaliénable, qui prime l'exigence du baptême, Thomas d'Aquin étend l'interdiction canonique des baptêmes forcés qui s'appliquait aux adultes. Le droit des parents en tant que tels subsistait en dépit de leur servitude, seulement « civile », qui n'excluait pas l'ordre du droit divin ou naturel, comme le précise Thomas d'Aquin dans la réfutation des objections ${ }^{36}$. Peut-être sa détermination comporte-t-elle en outre une dimension critique. Dans un contexte d'aggravation générale des dispositions antijuives, le rappel de la coutume de l'Église, conforté par l'invocation des « puissants princes catholiques » qui s'y étaient conformés de toute antiquité, marque une limite à la politique de Saint Louis en faveur des conversions. L'avertissement s'adresse également aux clercs qui, rompant avec les exemples de Sylvestre et d'Ambroise - dont Thomas endossait, en l'espèce, les fonctions de conseil -, eussent été mal avisés d'inciter le prince à enfreindre une norme rationnelle entérinée par l'usage.

Au milieu des années 1280, le franciscain Richard de Mediavilla reprend l'essentiel des arguments thomistes, sous une forme beaucoup plus ramassée ${ }^{37}$. Il assimile le droit des parents charnels à une possessio, à un

32. ARIStote, Éthique à Nicomaque, livre 5, 1134b 10-11; livre 8, 1161b18.

33. Thomas D’Aquin, Quodlibet II, 7, p. 223.

34. Ibid.

35. Ibid.

36. Ibid., p. 224.

37. Richard de Mediavilla, In IV Sent., D. 6, a. 3, q. 3, p. 78-79. 
dominium exercé sur le corps de leurs enfants et garanti par Dieu jusqu'à ce que ces enfants soient capables d'user de leur libre arbitre : «Bien que les enfants relèvent davantage du pouvoir de Dieu que de celui de leurs parents charnels, Dieu veut toutefois que les parents conservent intact le droit qu'ils possèdent sur le corps de leurs enfants. ${ }^{38}{ }^{2}$ En introduisant une comparaison entre pouvoir de Dieu et pouvoir des parents charnels sur l'enfant, Mediavilla ouvre cependant la voie à l'argumentaire spécifiquement politique développé par Duns Scot, qui débouche toutefois sur une conclusion rigoureusement opposée.

\section{Le conflit entre droit du prince et droit des parents comme sujets}

La position du franciscain anglais en faveur des baptêmes forcés marque une rupture nette au tournant du XIII et du XIV ${ }^{\mathrm{e}}$ siècles. Si l'argument suivant lequel on ne pouvait commettre l'injustice d'enlever leurs enfants aux juifs valait pour une personne privée, dit-il, il ne valait pas pour le prince, « dont les juifs sont les sujets dans le gouvernement de la chose publique. Car Dieu a sur le petit enfant un pouvoir supérieur à celui de ses parents. Or universellement, dans les puissances ordonnées, la puissance inférieure n'oblige pas à l'encontre de la puissance supérieure ${ }^{39} »$. En tant que serviteur de Dieu, le prince avait non seulement le pouvoir, mais le devoir (non solum licet, sed et debet) de faire enlever les enfants pour qu'ils soient baptisés et élevés dans le christianisme ${ }^{40}$. Qui plus est, il agirait « religieusement » en forçant les parents eux-mêmes à recevoir le baptême "par la menace et la terreur » ${ }^{41}$, à l'instar du « très religieux prince Sisebut ${ }^{42} »$. En prétendant lire, dans le canon De Iudeis du concile de Tolède IV inséré au Décret de Gratien, l'éloge de l'action du roi wisigoth qui avait ordonné la conversion générale des juifs de son royaume au $\mathrm{VII}^{\mathrm{e}}$ siècle, Duns Scot en détourne expressément le sens. Le canon rappelait l'action de Sisebut en interdisant de l'imiter.

Dans la hiérarchie des puissances invoquée par Duns Scot, le prince n'apparaît plus comme le relais des décisions de l'Église, mais comme l'intermédiaire direct du pouvoir divin. Cette hiérarchie ne ménage aucune place au pape, dont le rôle traditionnel de protecteur des juifs est totalement ignoré. Cette prise de position inhabituelle en faveur du pouvoir du prince en matière religieuse peut se lire comme une justification de l'expulsion des juifs d'Angleterre, ordonnée par Édouard I ${ }^{\mathrm{er}}$ au printemps 1290, après plus d'un demi-siècle d'efforts de conversion, minés, à partir des années 1280 ,

38. Ibid., p. 79.

39. Jean Duns Scot, Ordinatio in IV Sent., D. 4, q. 9, éd. cit. p. 58.

40. Ibid., p. 60.

41. Ibid.

42. Ibid., p. 61-62. 
par la crainte croissante de l'apostasie des convertis ${ }^{43}$. La question sur le baptême forcé pouvait prendre sens, chez Duns Scot, en fonction de cet événement. Si tous les sujets du prince chrétien devaient être chrétiens, l'expulsion était la seule issue s'ils persistaient à refuser le baptême ${ }^{44}$.

Thomas d'Aquin et Duns Scot ont ainsi situé le conflit de droits à des niveaux différents et l'ont résolu de manières radicalement divergentes. Pour Thomas d'Aquin, le droit naturel interdisait de séparer les jeunes enfants de leurs parents pour les faire baptiser. Pour Duns Scot, la hiérarchie des puissances imposait au contraire le rapt et le baptême des enfants juifs.

\section{Le conflit entre droit du prince et droit des parents comme esclaves}

De façon tout à fait frappante, les scolastiques qui ont traité la question du baptême forcé sous l'angle de la servitude des juifs ${ }^{45}$ ont envisagé cette servitude en termes strictement juridiques, sans jamais la relier explicitement à ses racines théologiques. L'idée selon laquelle la mort du Christ avait libéré les chrétiens et asservi les juifs n'est jamais rappelée dans la discussion. Les maîtres ont inscrit cette servitude dans la sphère du droit positif et se sont employés à en évaluer les effets juridiques, dans un contexte où cette forme de domination était du reste loin d'avoir disparu, comme l'a notamment souligné Laurent Feller ${ }^{46}$. Cela ne signifie pas que les juifs aient été réellement esclaves dans les sociétés chrétiennes des XIII ${ }^{\mathrm{e}}$ et $X^{\mathrm{e}}$ siècles. L'affirmation de la juridiction exclusive du souverain chrétien sur « ses juifs ${ }^{47}$ » donne toutefois lieu, sinon à un statut juridique formel, du

43. Cf. R. Stacey, "The Conversion of Jews to Christianity in Thirteenth Century England », Speculum, 67 (1992), p. 263-283 ; R. R. Mundill, England's Jewish Solution. Experiment and Expulsion, 1262-1290, Cambridge, 1998.

44. $C f$. E. Marmursztejn et S. Piron, « Duns Scot et la politique », p. 40-47.

45. Sur cette notion, on verra les articles classiques de S. Schwarzfuchs, « De la condition des juifs de France aux XII et XIII ${ }^{e}$ siècles ", Revue des études juives, 125 (1966), p. 221-232 ; S. W. BARON, « Medieval Nationalism and Jewish Serfdom », dans M. BENHorin, B. D. Weinryb, S. Zeituin éd., Studies and Essays in Honor of Abraham A. Neuman, Leyde, 1962, p. 17-48 ; ID., " "Plenitude of Apostolic Powers" and Medieval "Jewish Serfdom" », dans L. A. Feldman éd., Ancient and Medieval Jewish History. Essays by Salo Wittmayer Baron, New Brunswick, 1972, p. 284-307 ; G. LANGmUIR, «Tanquam servi: The Change in Jewish Status in French Law about $1200 »$, dans M. YARDENI éd., Les Juifs dans l'histoire de France, Leyde, 1980, p. 24-54, repris dans G. LANGMUIR, Towards a Definition of Antisemitism, Berkeley/Los Angeles, 1990, p. 167-194.

46. Cf. L. Feller, Paysans et seigneurs au Moyen Âge. VIII'-XV' siècles, Paris, 2007 (p. 166 sq.)

47. Sur les expressions, nombreuses, de cette revendication, on verra notamment $\mathrm{S}$. W. BARON, " Medieval Nationalism and Jewish Serfdom »... ; ID., " "Plenitude of Apostolic Powers"...»; A. Patschovsкy, "The Relationship between the Jews of Germany and the King (Eleventh-Fourteenth Centuries). A European Comparison », dans A. HaverKAMP et H. Vollrath éd., England and Germany in the High Middle Ages, Oxford, 1996, p. 193-218 ; G. LANGMUIR, «Tanquam servi...». 
moins à des incapacités et à des restrictions qui s'apparentent aux charges caractéristiques de la servitude, quelques variations qu'aient alors impliqué leurs traductions pratiques. Dans un ouvrage récent, Céline Balasse a montré que l'évolution politique des $\mathrm{XIII}^{\mathrm{e}}$ et $\mathrm{XIV}^{\mathrm{e}}$ siècles avait induit une dégradation sévère de la condition des juifs du royaume de France. Sous le règne de Philippe le Bel (1285-1314), les juifs n'ont plus aucune liberté de mouvement, leurs biens peuvent être taxés sans cause et leurs personnes données ou vendues ${ }^{48}$.

Les auteurs scolastiques qui ont envisagé le conflit de droits en postulant la servitude des juifs se sont focalisés sur les conséquences de la servitude en tant que telle. Elle fonctionne dans leurs discussions comme une catégorie générale pertinente pour penser l'absence ou la privation de droits. À cet égard, la question du baptême forcé des enfants juifs semble constituer un prolongement de la réflexion sur la possibilité de dissoudre les unions serviles, que les scolastiques avaient conduite à partir de la question de savoir si l'esclave était soumis à son maître dans ce qui relevait des nécessités naturelles liées à la conservation de soi et à celle de l'espèce. Les réponses des théologiens sont généralement négatives. Le conflit entre droits du maître et droits des esclaves débouchait sur la délimitation de zones franches dans lesquelles les esclaves étaient libres de jouir de leurs droits naturels, nonobstant les droits de leur maître.

$\mathrm{Au}$ début du XIV ${ }^{\mathrm{e}}$ siècle, Durand de Saint-Pourçain est le premier à avoir envisagé la question du baptême forcé sous l'angle des effets juridiques de la servitude des juifs. Thomas d'Aquin avait certes introduit le motif, mais il avait limité son traitement à une brève réfutation, consécutive à la responsio proprement dite. Durand est en outre le seul à avoir pris position en faveur du baptême forcé des enfants juifs sur ce terrain. Sa détermination se fonde sur le principe suivant lequel le prince chrétien pouvait licitement enlever les enfants des juifs pour les vendre, comme n'importe quel bien ${ }^{49}$. Leur acquéreur pouvait donc en disposer à sa guise et les faire baptiser. Durand de Saint-Pourçain prend toutefois soin de distinguer le cas des enfants, «privés de l'usage de la raison » et qui tireraient en outre un « grand bénéfice » du baptême, du cas des « juifs et des autres infidèles adultes » qui jouissaient, en droit naturel, d'une liberté de croire équivalente à celle de manger, de boire ou de se marier ${ }^{50}$.

Dans son commentaire sur les Sentences, Pierre de la Palud répond que la propriété n'autorise pas tous les usages ${ }^{51}$. Une esclave était destinée au service de son maître, non à ses plaisirs ; elle ne pouvait être contrainte

48. Cf. C. Balasse, 1306. L'Expulsion des juifs du royaume de France, Bruxelles, 2008, p. 47.

49. Durand de Saint-Pourçain, In IV Sent., lib. 4, D. 4, q. 6, f. 260r.

50. Ibid.

51. Pierre de la Palud se réfère implicitement à un principe de droit romain, invoqué par ailleurs par Innocent IV à l'encontre de la séparation des époux de statut servile ; 
de se marier contre son gré. De même, bien que le juif appartienne au prince (quamvis sit res sua), il ne pouvait être contraint au baptême, ni en lui-même, ni en la personne de son fils ${ }^{52}$. La distinction qu'avait opérée Durand de Saint-Pourçain entre le régime des adultes et celui des enfants est supprimée au moyen de la notion de « volonté interprétative » : «Seule la volonté des parents est la volonté interprétative des jeunes enfants avant qu'ils aient l'usage de la raison ${ }^{53} »$. Cet argument imposait des limites strictes à « l'ordre du droit humain dont [relevait] la servitude ${ }^{54} »$.

Guido Terreni étoffe la liste des droits naturels de l'esclave dans son commentaire sur le Décret de Gratien, en y ajoutant le droit d'éduquer ses enfants ${ }^{55}$. Le maître ne pouvait vendre l'enfant, parce que l'enfant ne pouvait être séparé de ses parents tant qu'il n'avait pas l'usage de la raison. Cet argument se fonde sur une analogie qui indique le lien avec le débat sur les rapports entre mariage et servitude : le maître n'avait pas plus le droit de séparer les enfants de leurs parents qu'il n'avait le droit de vendre séparément des esclaves unis par mariage, " en raison du devoir, de droit divin et naturel, par lequel l'homme et son épouse sont une seule chair et sont tenus de se rendre mutuellement le devoir [conjugal] ; et c'est pourquoi ils doivent rester attachés l'un à l'autre ${ }^{56} »$. Après avoir balayé les conclusions de Durand de Saint-Pourçain, Guido Terreni entreprend de réfuter l'argument scotiste en vertu duquel le prince « doit maintenir le droit et le pouvoir de Dieu (ius et dominium Dei), qui est un seigneur supérieur aux parents ${ }^{57}$ » : « Ni dans l'Ancien, ni dans le Nouveau Testament », dit Guido Terreni, « Dieu n'ordonne que les enfants soient enlevés à leurs parents. Il ordonne au contraire qu'on n'enlève à personne ce qui lui appartient et qu'on ne fasse injustice à quiconque. Or en vertu du droit divin ou naturel, en ce qui concerne l'éducation, les enfants appartiennent à leurs parents (sunt res parentum) et non aux princes. Ils ne peuvent donc leur être enlevés sans injustice $»^{58}$. Guido Terreni conclut que le prince « ne doit chercher à accomplir le droit et le pouvoir de Dieu qu'autant que Dieu le veuille ; or Dieu ne veut pas être servi à contre-cœur (invite et involuntarie) $)^{59} »$.

cf. Digeste 1, 6, 2, éd. Th. Mommsen, Berlin, 1895, p. 8 ; InNocent IV, Apparatus in quinque libros decretalium, Francfort, 1570, f. 473v : «Interest enim, ne quis re sua male utatur ».

52. Pierre de la Palud, In IV Sent., lib. 4, D. 4, q. 4, a. 1, f. 20 r.

53. Ibid.

54. Ibid.

55. Guido Terreni, Apparatus in Decretum Gratiani (Paris, BnF, ms. lat. 3914, f. 328v).

56. Ibid.

57. Ibid., f. 329v.

58. Ibid.

59. Ibid. 
Loin d'avoir produit des réponses convenues sur l'exigence universelle du baptême, les auteurs scolastiques ont résolu de façons très différentes une question qu'ils ont envisagée dans les termes d'un conflit de droits entre prince chrétien et parents juifs. La focalisation de la discussion sur ce conflit de droits résulte du glissement de la question du baptême vers celle de l'enlèvement des enfants juifs. Induit par la prise en compte de la spécificité de l'enfance, ce déplacement témoigne aussi de l'ancrage de la pensée scolastique en son temps. La question émerge dans des contextes politiques et juridiques qui déterminent en partie l'orientation et les développements singuliers de son traitement. De fait, les textes scolastiques font varier les termes du conflit de droits. Le droit du prince est référé par Thomas d'Aquin et Richard de Mediavilla à la coutume de l'Église, par Duns Scot au droit de Dieu sur l'enfant, par Durand de Saint-Pourçain, Pierre de la Palud et Guido Terreni au droit du maître sur ses esclaves. Le droit des juifs est déduit de leur sujétion ou, plus souvent, de leur servitude. Aussi la question du baptême forcé des enfants juifs n'est-elle exiguë qu'en apparence. Elle participe d'une réflexion sur l'étendue et sur les limites de la domination chrétienne sur les juifs comme parents, comme sujets ou comme esclaves. La mise à l'épreuve des liens de filiation naturelle ne s'inscrit pas fortuitement dans ce cadre. Les enjeux politiques des questions relatives à la filiation, fertiles en conflits de normes structurellement dominés par l'opposition entre la nature et le droit, s'observent dans la longue durée. D'autant plus aigu que les droits créés par la filiation naturelle s'y heurtent aux exigences de la Loi nouvelle elle-même, le conflit de droits construit par les scolastiques à partir de la question du baptême forcé des enfants juifs l'inscrit pleinement dans l'histoire politique des relations entre juifs et chrétiens au Moyen Âge.

Elsa Marmursztejn - Université de Reims - CERHIC EA 2616

\section{Conflits de normes dans le débat scolastique sur le baptême forcé des enfants juifs (XIII'-XIV ${ }^{\mathrm{e}}$ siècle)}

Successivement traitée, entre 1269 et 1340, par Thomas d'Aquin, Richard de Mediavilla, Jean Duns Scot, Durand de Saint-Pourçain, Pierre de la Palud et Guido Terreni, la question du baptême forcé des enfants juifs révèle la pertinence et la productivité des conflits de normes dans le champ du débat théologique. La discussion ne s'est pas portée sur le terrain "légal " de l'opposition entre christianisme et judaïsme, mais sur le terrain politique et juridique du conflit entre droits du prince chrétien et droits des juifs comme parents, comme sujets et comme esclaves, dans un contexte où les politiques de conversion contribuent à la définition des monarchies occidentales comme monarchies chrétiennes et où la revendication royale d'une juridiction exclusive sur les juifs participe de l'affirmation générale de la souveraineté. Les liens de filiation naturelle sont ainsi mis à l'épreuve dans le cadre d'une réflexion plus large sur l'étendue et les limites de la domination chrétienne.

Scolastique - baptême forcé - enfants juifs - sujétion - servitude des juifs 


\section{Conflicts of Norms in Scholastic Debates on Forced Baptism of Jewish Children (Thirteenth-Fourteenth Century)}

Between 1269 and 1340, Thomas Aquinas, Richard of Mediavilla, John Duns Scotus, Durandus of Saint-Pourçain, Pierre de la Palud, and Guido Terreni successively dealt with the question of the forced baptism of Jewish children, which shows the relevance and the productivity of conflicts of norms in the field of theological debate. The discussion has not been brought to the "legal " ground of the opposition between Christinanity and Judaism, but to the political and juridical ground of a conflict between the rights of the Christian ruler and those of the Jews as parents, as subjects, and as slaves, in a context in which the conversionist policies contributed to the definition of Western monarchies as Christian monarchies, and in which the royal claim to an exclusive jurisdiction over the Jews participates in the global affirmation of sovereignty. Thus, the links of natural filiation are put to trial within the frame of a larger reflexion about the extension and the limits of Christian rulership.

Scholasticism - forced baptism - Jewish children - subjection - Jewish serfdom 